\title{
IMPACTOS DA POLÍTICA NACIONAL DE RESÍDUOS SÓLIDOS NA QUALIDADE DE VIDA: UMA AVALIAÇÃO NOS MUNICÍPIOS MINEIROS ${ }^{1}$
}

\author{
Loredany Consule Crespo Rodrigues ${ }^{2}$ \\ Cristiana Tristão Rodrigues ${ }^{3}$
}

\begin{abstract}
Considerando a relevância das questões ambientais e os efeitos negativos causados pela má gestão dos resíduos sólidos ao meio ambiente, o objetivo deste trabalho é avaliar os impactos da Política Nacional de Resíduos Sólidos (PNRS), instituída pela Lei no 12.305/2010, sobre a existência de lixões, o número de famílias vulneráveis e a proporção de internações relacionadas ao saneamento básico inadequado nos municípios de Minas Gerais. Para atingir este objetivo, foi utilizado como método de análise estatística o propensity score matching (PSM) e dados referentes ao ano de 2013. Os resultados encontrados revelam que, embora os impactos da política em relação à redução do número de lixões e do número de famílias que se encontram em situação de vulnerabilidade não tenham sido estatisticamente significativos, a proporção de internações relacionadas ao mau saneamento nos municípios que implementaram o Plano Municipal de Gestão Integral de Resíduos Sólidos (PMGIRS), conforme exigências da PNRS, é menor que nos municípios que ainda não possuem o plano. Conclui-se, então, que a PNRS tem gerado impactos positivos na saúde da população, melhorando a qualidade de vida, podendo afetar, inclusive, a formação de capital humano destes municípios.
\end{abstract}

Palavras-chave: avaliação de políticas públicas; política nacional de resíduos sólidos; bem-estar; propensity score matching.

\section{THE IMPACTS OF THE NATIONAL SOLID WASTE POLICY ON THE QUALITY OF LIFE: AN EVALUATION IN MUNICIPALITIES OF MINAS GERAIS}

Considering the relevance of environmental issues and the impacts that solid waste causes to the environment, the present study aim to evaluate the effect of the PNRS, instituted by Law $n$. $12.305 / 2010$, on the existence of landfills and the impact on the number of vulnerable families and the proportion of hospitalizations related to inadequate sanitation in the municipalities of Minas Gerais. To achieve this goal, propensity score matching and data referring to the year 2013 were used. The results found reveal that, although the impact of the policy in relation to the persistence of dumps and families under vulnerability was not statistically significant, the proportion of hospitalizations related to poor sanitation in treated municipalities is lower than in control municipalities. It is then concluded that the PNRS has generated positive impacts on the health of the population, improving the quality of life, and can affect, even, the human capital formation of these municipalities.

Keywords: evaluation of public policies; national solid waste policy; welfare; propensity score matching.

1. DOI: http://dx.doi.org/10.38116/ppp56art4

2. Doutoranda em economia aplicada no Departamento de Economia Rural na Universidade Federal de Viçosa (DER-UFV).

E-mail:<loredanyufv@hotmail.com>.

3. Professora adjunta do Departamento de Economia (DEE) da UFV. E-mail: <cristiana.rodrigues@ufv.br>. 


\section{LOS IMPACTOS DE LA POLÍTICA NACIONAL DE RESIDUOS SÓLIDOS EN LA CALIDAD DE VIDA: UNA EVALUACIÓN EN LOS MUNICIPIOS MINEROS}

Teniendo en cuenta la relevancia de las cuestiones ambientales y los impactos que los residuos sólidos causan al medio ambiente, el objetivo del presente trabajo es evaluar el efecto de la PNRS, instituida por la Ley n. 12.305/2010, sobre la existencia de basurales y el impacto sobre el número de familias vulnerables y la proporción de internaciones relacionadas con el saneamiento básico inadecuado en los municipios de Minas Gerais. Para alcanzar este objetivo se utilizó el índice de ponderación y los datos correspondientes al año 2013. Los resultados encontrados revelan que, aunque el impacto de la política en relación con la persistencia de basurales y familias en vulnerabilidade no fue estadísticamente significativo, la proporción de internaciones relacionadas con el mal saneamiento en los municipios tratados es menor que en los municipios de control. Se concluye entonces que la PNRS ha generado impactos positivos en la salud de la población, mejorando la calidad de vida, pudiendo afectar, inclusive, la formación de capital humano de estos municipios.

Palabras clave: evaluación de políticas públicas; política nacional de residuos sólidos; bienestar; propensity score matching.

\section{LES IMPACTS DE LA POLITIQUE NATIONALE SUR LES DÉCHETS SOLIDES SUR LA QUALITÉ DE VIE: UNE ÉVALUATION DANS LES MUNICIPALITÉS DE MINAS GERAIS}

Considérant la pertinence des questions environnementales et les impacts que les déchets solides causent sur l'environnement, I'objectif de la présente étude est d'évaluer l'effet du PNRS, institué par la Loi n. 12.305/2010, sur l'existence de décharges et l'impact sur le nombre de familles vulnérables et la proportion d'hospitalisations liées à un assainissement insuffisant dans les municipalités de Minas Gerais. Pour atteindre cet objectif, nous avons utilisé l'appariement par score de propension et les données se rapportant à l'année 2013. Les résultats trouvés révèlent que, même que l'impact de la politique sur la persistence des décharges et des familles en situation de vulnérabilité n'était pas statistiquement significatif, la proportion d'hospitalisations l'assainissement dans les municipalités traitées est plus faible que dans les municipalités contrôlées. Il est alors concluil que le PNRS a généré des impacts positifs sur la santé de la population, améliorant la qualité de vie, et pouvant affecter, inclus, la formation du capital humain de ces municipalités.

Mots-clés: evaluation des politiques publiques; politique nationale des déchets solides; bien-être; appariement de score de propension.

JEL: Q58; K32; 131.

\section{INTRODUÇÃO}

As mudanças climáticas e os desastres naturais cada vez mais frequentes em todo o mundo têm despertado o interesse de governantes. Considerando que os danos ambientais ultrapassam fronteiras, há uma busca para manter o desenvolvimento dos países, mas de forma sustentável.

Um dos maiores problemas enfrentado é o aumento significativo dos resíduos sólidos, que está diretamente relacionado com a participação humana (Okada, 2012). 
Para Jacobi e Besen (2011), o consumo excessivo e supérfluo e os modelos produtivos baseados na obsolescência programada e descartabilidade dos produtos têm aumentado significativamente a geração de resíduos sólidos. Consequentemente, os impactos causados ao meio ambiente pela má gestão e pelo destino inadequado desses resíduos são cada vez maiores.

Diante disso, inúmeros países têm se esforçado e se unido por meio de diversas conferências e acordos mundiais como a Rio 92, o Protocolo de Quioto, a Rio+20, o Acordo do Clima de Paris, entre outros, em busca de minimizar tais impactos, demonstrando assim a relevância do assunto mundialmente. Diante de fenômenos como o efeito estufa e o aquecimento global, essas conferências e esses acordos buscam mostrar a importância do assunto e a necessidade do comprometimento de todos os países para minimizar esses impactos, visando à manutenção das condiçóes necessárias para a vida humana.

A redução de resíduos nas fontes geradoras; a diminuição da disposição final no solo; a maximização do reaproveitamento, da coleta seletiva e da reciclagem, com inclusáo socioprodutiva de catadores e participaçáo da sociedade; a compostagem e a recuperação de energia se tornaram prioridades para a atuação dos governos (Jacobi e Besen, 2011).

O consumismo e o progresso tecnológico tornam o problema ainda maior, pois reduz a vida útil dos produtos que, muitas vezes, são descartados em plena condição de uso. De acordo com Sette e Nogueira (2010), o aumento dos resíduos tem sido impactado pelo aparecimento de grandes cidades, crescimento populacional, consumo exponencial na busca de satisfação de desejos humanos ilimitados e corrida do setor produtivo para atendê-los. Porém, conforme Arcila (2008), esse aumento não tem sido acompanhado pelo cuidado com sua disposição final.

Segundo Besen et al. (2010), os impactos socioambientais causados pela gestão e disposição inadequadas dos resíduos sólidos são diversos: degradação do solo, comprometimento dos corpos d'água e mananciais, intensificação de enchentes, contribuição para a poluição do ar, proliferação de vetores de importância sanitária nos centros urbanos e catação em condições insalubres nas ruas e nas áreas de disposição final.

Para Gouveia (1999), se não houver controle sobre os gases tóxicos liberados no processo de decomposição orgânica do lixo, os resíduos podem contaminar o solo e os lençóis freáticos.

O problema se torna ainda maior quando parte da população mais vulnerável encontra nos lixóes uma "oportunidade" de aquisição de renda, por meio da coleta de materiais que podem ser reaproveitados e reciclados. Tendo em vista que os lixôes são ambientes propícios para a reprodução de vetores entre outros elementos 
patológicos, esses catadores colocam em risco sua saúde física ao frequentarem esse ambiente (Monteiro et al., 2001).

Observa-se, portanto, que os efeitos negativos provenientes dos resíduos sólidos não se limitam ao meio ambiente, mas impactam a saúde individual e coletiva, tornando ainda mais relevante a elaboração de políticas públicas que incentivem sistemas de coleta e disposição final desses resíduos de forma adequada, incluindo a proteção necessária à saúde desses trabalhadores (Ferreira e Anjos, 2001).

Nesse contexto, a Política Nacional de Resíduos Sólidos (PNRS) surge como uma forma de conscientização e envolvimento de diversos atores sociais na busca pela minimização dos problemas causados pela gestão e pelo tratamento inadequados dos resíduos sólidos no país. A PNRS, Lei no 12.305 , de 2 de agosto de 2010, demonstra o comprometimento do governo e, consequentemente, do país frente às questóes ambientais.

A PNRS tem como alguns de seus instrumentos a coleta seletiva, a logística reversa, a educação ambiental e os planos de resíduos sólidos. O programa visa à participação de todas as esferas (federal, estadual e municipal; pública e privada), a fim de tornar-se mais eficaz.

Nesse sentido, esta pesquisa tem como objetivo avaliar, por meio da metodologia propensity score matching (PSM), o efeito da PNRS nos municípios que implementaram os Planos Municipais de Gestão Integrada de Resíduos Sólidos (PMGIRS) segundo as exigências da PNRS, em relação à redução dos lixóes, assim como os impactos no número de famílias vulneráveis e na taxa de internações decorrentes do saneamento inadequado.

Desde sua implementação, em 2010, a PNRS foi avaliada em diferentes aspectos. Alencar, Rocha e Silva (2015) analisaram as consequências da implementação da PNRS a partir do estudo de caso de cooperativas dos municípios fluminenses. Sousa e Fernandes (2016) investigaram os impactos ambientais decorrentes do lixão no município de Imperatriz-MA, verificando a importância de o município se adequar às exigências da PNRS em relação à gestão e ao gerenciamento dos resíduos. No mesmo ano, Silva e Teixeira (2016) avaliaram a PMGIRS de Barreiras-BA, enfatizando a coleta seletiva.

Um ano mais tarde, Silva, Fugii e Santoyo (2017) aplicaram um modelo de avaliação da gestão integrada de resíduos sólidos ao plano municipal de Curitiba; e Castro, Souza e Albino (2017) investigaram o impacto da PNRS sobre a coleta seletiva dos resíduos sólidos urbanos (RSU) em Viçosa-MG.

Diferentemente do que foi realizado até o momento, este trabalho examina os impactos da política na qualidade de vida e no bem-estar da população mineira. Vale ressaltar, que a avaliação de políticas públicas é de suma importância para um 
país que tem como objetivo aprimorar constantemente seu ciclo de políticas. Esse processo de avaliação possibilita a identificação de falhas e auxilia na elaboração de políticas cada vez mais eficientes e eficazes. Sendo assim, tornam-se cada vez mais significativas pesquisas relacionadas ao tema.

$\mathrm{O}$ artigo está estruturado em mais quatro seções, além desta introdução. $\mathrm{Na}$ seção 2, é apresentado o referencial teórico acerca do ciclo de políticas públicas (policy cycle) e a PNRS; em seguida, na seção 3, encontram-se as principais ferramentas metodológicas utilizadas na análise, assim como os dados e as variáveis utilizados. Na seção 4, os resultados são apresentados e discutidos e, por fim, na seção 5 , o trabalho é concluído.

\section{CICLO DE POLÍTICAS PÚBLICAS (POLICY CYCLE)}

Considerando que a política pública é aquela proveniente do Estado, ou seja, são decisôes tomadas por agentes governamentais baseadas na autoridade soberana do poder público, a PNRS é uma política pública direcionada para a gestão e o tratamento de resíduos sólidos. Na literatura, há diversos conceitos de política pública. Para Lynn (1980), esta política é um conjunto de ações governamentais que produzirá efeitos específicos. Para Peters (1986), trata-se da soma das atividades do governo, que atua de forma direta ou por delegação, e influencia a vida dos cidadáos. Segundo Mead (1995), a política pública é um campo no estudo da política que analisa as ações do governo à luz de grandes questóes públicas. Outra definição encontrada na literatura é a de que decisões e análises sobre política pública implicam responder às questôes: quem ganha o quê, por quê e que diferença faz (Laswell, 1936).

De acordo com Easton (1970), uma política pública é resultante do processamento, pelo sistema político, de inputs e withinputs. Enquanto os primeiros são as demandas originadas do meio ambiente, isto é, expressam demandas e apoios provenientes de cidadãos que estão fora do sistema político; os withinputs são demandas originadas no próprio sistema político, ou seja, são provenientes de agentes do Executivo, parlamentares, governadores, ou do Judiciário.

Para que uma política pública seja implementada, há diversas etapas percorridas, denominado na literatura por "ciclo de políticas" (policy cycle). Este ciclo é composto pelas seguintes etapas: i) identificação do problema; ii) formação da agenda; iii) formulação de alternativas ou da política; iv) tomada de decisão; v) implementação; e vi) avaliação e extinção. Vale ressaltar que embora este ciclo divida a política pública em etapas sequenciais, ela ocorre de forma dinâmica e sistêmica, o que torna sua avaliação ainda mais complexa.

De acordo com Sjoblom (1984), a fase de identificação envolve a percepçáo, a definição e delimitação do problema e a avaliação da possibilidade de resolução. 
Ou seja, o que era um "estado de coisas" passa a ser um problema político. A formação da agenda ocorre quando o problema político passa a ser relevante, ou seja, passa a ser discutido por um grupo de autoridades dentro e fora do governo. Segundo Cobb e Elder (1983), para que um problema entre na agenda política, é necessário que diferentes atores entendam que determinada situação precisa de intervenção; que as açôes propostas são factíveis e irăo solucionar o problema; e que o problema toque as responsabilidades públicas.

A partir da agenda formada, buscam-se alternativas a fim de solucionar o problema (formulação da política). Conforme Schattschneider (1960, p. 68), "a definição de alternativas é o instrumento supremo de poder, porque a definição de alternativas é a escolha dos conflitos, e a escolha dos conflitos aloca poder". Após a formulação de alternativas, inicia-se a etapa de tomada de decisão que decide qual a melhor alternativa para atender e solucionar o problema. Nesta etapa, há presença de um jogo político, em que os atores mobilizam todos os seus recursos de poder em busca de que a proposta de seu interesse seja a escolhida.

Com a decisão tomada, implementa-se a política. Este é o momento em que as regras, as rotinas e os processos sociais propostos tornam-se açóes (O’Toole Junior, 2003). Nesta etapa, a política é de fato colocada em prática e começa a ter resultados, permitindo sua avaliação. Vale ressaltar que, segundo Anderson (1979), a avaliação de políticas públicas não ocorre somente em relação aos resultados da implementação, isto é, se os projetos colocados em prática obtiveram ou não sucesso, mas também envolve o processo de julgamento sobre a validade das propostas para a ação pública (a política). De outro modo, a avaliação pode ser $e x$ ante (anterior à implementação) ou ex post (posterior à implementação). Segundo Costa e Castanhar (2003), existe a avaliaçáo in itinere, que ocorre no processo de implementação da política para ajustes imediatos.

A última etapa, denominada extinção, ocorre quando a política deixa de existir ou é substituída. Conforme Giuliani (2005), uma política pode ser extinta por três motivos: o problema que a originou foi solucionado; a política não está sendo eficaz; ou o problema ainda existe mas deixou de fazer parte da agenda governamental, ou seja, deixou de receber a atenção dos políticos.

A avaliação de políticas públicas é de suma importância, pois permite verificar a eficiência, a eficácia, os efeitos e os impactos causados por sua implementação. Esta é a etapa em que se encontra a PNRS, uma política que está vigente desde 2010.

Nos países democráticos do Ocidente, a avaliação de políticas públicas ganhou relevância, inclusive na América Latina, a partir da década de 1990. Segundo Faria e Filgueiras (2007), a avaliação foi colocada a serviço da reforma do setor público, que é submetido a reformas de diversas intensidades. Porém, ainda conforme os autores, no início do boom de avaliação de políticas públicas, que ocorreu na década 
de 1960 nos Estados Unidos, tinha-se a visão de que a avaliação era, basicamente, uma ferramenta de planejamento destinada aos formuladores de políticas e aos gerentes de escalóes superiores.

De acordo com Guba e Lincoln (1989), o início da avaliação de políticas públicas era profundamente técnico. A partir da Segunda Guerra Mundial até meados da década de 1960, passou a ser mais descritiva. De meados desta década até meados da década seguinte, prevaleceu uma fase que enfatizava a formulação de juízo de valor e, posteriormente, surgiu uma geração reativa, no sentido de reagir aos resultados e benefícios das políticas públicas.

Conforme Ham e Hill (1993), as análises de política são classificadas em duas categorias: a analysis of policy que visa, basicamente, ao melhor entendimento do processo político, tendo em vista que produz conhecimento sobre todo o processo da política (da formulação até a avaliação) e a analysis for policy, que envolve diretamente a tomada de decisão, isto é, refere-se à atividade aplicada voltada à solução de problemas sociais. Ou seja, enquanto a primeira tem um caráter descritivo, a segunda, um caráter prescritivo. Deste modo, a análise de política pública tem objetivo de melhorar o entendimento da política e do processo político, assim como apresentar propostas para o seu aperfeiçoamento.

Sendo assim, avaliar políticas públicas é importante para auxiliar no seu aperfeiçoamento, identificando as falhas e os sucessos, além de criar alternativas para o seu aprimoramento, assim como verificar os efeitos e impactos causados após sua implementação.

\subsection{PNRS e o ciclo de política (policy cycle)}

A PNRS surgiu como uma política pública cujos objetivos estáo diretamente relacionados com questóes ambientais que, atualmente, fazem parte da agenda política de governantes mundiais. A política tem como um de seus objetivos não gerar, reduzir, reutilizar, reciclar e tratar dos resíduos sólidos, bem como realizar a disposição final ambientalmente adequada dos rejeitos (Brasil, 2010a).

Cabe destacar que as questôes relacionadas ao tratamento de resíduos sólidos no Brasil podem ser consideradas uma demanda recorrente, haja vista que, conforme Ministério do Meio Ambiente (MMA), desde 1991, tramitava no Congresso Nacional um Projeto de Lei (PL) no 203/1991, que dispunha sobre o acondicionamento, a coleta, o tratamento, o transporte e a destinaçấo final dos resíduos de serviços de saúde.

A partir de 2004, questôes relacionadas aos resíduos sólidos passam a ser prioridade na agenda governamental, sendo instituído, inclusive, um grupo de discussão interministerial sobre o assunto. O MMA passa a concentrar esforços a 
fim de elaborar uma proposta que criasse diretrizes gerais aplicáveis aos resíduos sólidos do país.

É importante dizer que os diversos atores envolvidos com a gestão de resíduos sólidos participaram da formulação da política. Após diversas discussóes, realizadas pelo grupo interno do MMA, foi elaborado um anteprojeto de lei da Politica Nacional de Resíduos Sólidos que, além de ter sido debatido entre todos os ministérios relacionados com o tema, foi discutido pela sociedade por meio de seminários promovidos pelo MMA, pelos Ministérios das Cidades (MCidades) e da Saúde (MS); pela Fundação Nacional de Saúde (Funasa) e pela Caixa Econômica Federal (Caixa) (Brasil, [s.d.]).

Essa interação é de suma importância na fase de formulação da política, pois permite que questôes, muitas vezes não observadas pelos formuladores, sejam expostas pela sociedade que conhece a realidade do local onde a política será implementada.

Em dezembro de 2005, uma nova proposta, resultante das discussóes entre os diversos atores envolvidos, foi encaminhada à Casa Civil e aprovada em julho de 2006 pela comissáo especial criada para avaliar este PL. Porém, diversas questóes discutidas pelo governo federal junto à sociedade e ao setor produtivo não foram consideradas.

Dessa forma, em setembro de 2007, o governo federal encaminhou um anteprojeto à Câmara dos Deputados (PL no 1991/2007) que, além de manter a integraçáo dos catadores, consolidou o conceito de "logística reversa" (Castro, 2012).

Em junho de 2009, após diversas audiências públicas, visitas, debates e reuniôes técnicas externas, apresentou-se a Minuta de subemenda substitutiva global de plenário ao PL no 203/1991 e seus apensos, cuja aprovação ocorreu em março de 2010 pelo plenário da Câmara. Em julho de 2010, o texto foi aprovado pelo Senado Federal com pequena alteração e, em agosto do mesmo ano, foi sancionado pela Presidência da República, sem nenhum veto. Consequentemente, a Lei no 12.305 , de 2 de agosto de 2010, que instituiu a PNRS, foi publicada no Diário Oficial da Uniâo e regulamentada em dezembro de 2010 por meio do Decreto no 7.404/2010 (Brasil, [s.d.]).

Nesse contexto, ressalta-se a importância da participação e do interesse dos governantes para que uma demanda chegue à agenda governamental e se torne uma política pública. De acordo com Costa e Melo (1998, apud Pinto, 2008), para que um problema seja reconhecido e incorporado na agenda política, é necessário que o governo "acate" determinado assunto, permitindo que ele chegue ao debate público, gerando opçôes de políticas públicas. Isso é claramente observado neste 
caso, em que a demanda existia desde 1991 e a política foi implementada quase vinte anos mais tarde, em 2010.

A PNRS tem como alguns de seus objetivos a proteção da saúde pública e da qualidade ambiental; a não geração, redução, reutilização, reciclagem e tratamento dos resíduos sólidos, bem como a disposição final ambientalmente adequada dos rejeitos; a gestão integrada de resíduos sólidos; a integração dos catadores de materiais reutilizáveis e recicláveis nas açôes que envolvam a responsabilidade compartilhada pelo ciclo de vida dos produtos, entre outros (Brasil, 2010a).

Outra característica da PNRS é envolver as esferas pública e privada na gestão dos resíduos sólidos. Conforme o art. 4:

A Política Nacional de Resíduos Sólidos reúne o conjunto de princípios, objetivos, instrumentos, diretrizes, metas e açóes adotadas pelo Governo Federal, isoladamente ou em regime de cooperação com Estados, Distrito Federal, Municípios ou particulares, com vistas à gestáo integrada e ao gerenciamento ambientalmente adequado dos resíduos sólidos (Brasil, 2010a, art. 4º).

Nota-se a relevância da PNRS que, a partir de suas metas e seus objetivos, envolvendo diversas esferas de poder, traz benefícios não só para o meio ambiente, mas também para toda a sociedade que usufruirá de melhores condiçóes de vida a partir do tratamento adequado dos resíduos sólidos e das oportunidades geradas para os catadores. A política prioriza o desenvolvimento sustentável: ambientalmente correto, economicamente viável e socialmente justo, tornando sua avaliação ainda mais relevante, podendo, inclusive, auxiliar na elaboraçáo e formulação de políticas futuras.

\section{METODOLOGIA}

Um dos maiores problemas encontrados na avaliação de políticas públicas está relacionado à impossibilidade de se comparar um mesmo indivíduo em duas situaçóes distintas (participou e não participou da política), justamente por não possuir os dados do indivíduo que participou, caso não tivesse participado (Wooldridge, 2000; 2002).

Nesse sentido, busca-se encontrar um grupo de controle que seja comparável ao grupo de tratamento, podendo ser utilizado como contrafactual (Wooldridge, $2000 ; 2002)$. Busca-se, assim, por um grupo de municípios que náo implementou a política, mas que seja o mais semelhante possível do grupo que a implementou, podendo ser comparados. E um dos métodos utilizados para realizar este pareamento é o propensity score matching (PSM). 


\subsection{Propensity score matching}

Apesar de a implementação da PNRS ser aleatória, é possível que algumas características dos municípios restrinjam sua efetiva participação, limitando, inclusive, a criação da PMGIRS nos termos estabelecidos pela lei. Neste sentido, a comparação direta dos municípios tratados e de controle pode levar a resultados viesados, isto é, que não expressam o real efeito da política.

Dessa forma, optou-se por utilizar o PSM, que é capaz de corrigir o possível viés existente (Rosenbaum e Rubin, 1983), para identificar o efeito da política. Esse método busca, a partir de um vetor de características observáveis, identificar um grupo de comparação o mais semelhante possível do grupo de tratamento (participantes) e, ao comparar os resultados de ambos os grupos, após a implementação da política, é possível reconhecer seus efeitos e impactos.

Inicialmente, estima-se uma regressão de probabilidade em que, a partir de características observáveis, identifica-se a probabilidade de participação no programa. As variáveis explicativas da regressáo devem ser selecionadas de acordo com a sua influência na probabilidade de implementar a política, como a existência de legislação específica para o meio ambiente e de conselho municipal de meio ambiente e nas variáveis resultado que, no caso deste estudo, são o número de famílias vulneráveis, a proporção de internaçóes por doenças relacionadas ao saneamento ambiental inadequado e a existência de lixôes no município.

Desse modo, define-se o PSM como a probabilidade condicional de receber o tratamento, dado o vetor de características observáveis (Rosenbaum e Rubin, 1983). Ou seja:

$$
\mathrm{P}(\mathrm{X})=\operatorname{Pr}(\mathrm{D}=1 \mid \mathrm{X}),
$$

em que, a variável dependente $(\mathrm{P}(\mathrm{X})$ ) corresponde à probabilidade de implementar a política; $D$ é uma variável binária (igual a 1 se o município implementou a PMGIRS e, 0 caso contrário) e; $X$ corresponde ao vetor de características observáveis que podem afetar, de alguma forma, a implementação da política.

Formalmente, para o caso específico deste estudo, tem-se que:

$$
\begin{aligned}
\text { PMGIRS }_{i}= & \beta_{0}+\beta_{1} \text { leg }_{i}+\beta_{2} \text { pbf }_{i}+\beta_{3} \text { emp }_{i}+\beta_{4} p s f_{i}+\beta_{5} \text { cmma }_{i}+\beta_{6} \text { pop }_{i} \\
& +\beta_{7} \text { urb }_{i}+\beta_{8} \text { pibpc }_{i}+\beta_{9} \text { ete } \_50_{i}+\beta_{10} \text { tratlix }_{-} 70_{i}+\varepsilon_{i}
\end{aligned}
$$

A descrição das variáveis utilizadas nesta etapa é apresentada no quadro 1. 
QUADRO 1

Variáveis utilizadas no modelo probit

\begin{tabular}{|c|c|}
\hline Variáveis & Descrição \\
\hline pmgirs & $\begin{array}{l}\text { Existência de política municipal de gestão integrada de resíduos sólidos, conforme termos estabelecidos } \\
\text { pela PNRS. Trata-se da variável dependente do modelo e é representada por uma dummy, que recebe valor } \\
\text { unitário caso exista a política no município e, zero, caso contrário. }\end{array}$ \\
\hline pop & População do município, em número de habitantes. \\
\hline pibpc & $\begin{array}{l}\text { Produto interno bruto (PIB) per capita, composto pela soma do valor agregado bruto de todos os residentes, } \\
\text { dividido pela população do município, em } \mathrm{R} \$ \text {. }\end{array}$ \\
\hline $\mathrm{cmma}$ & $\begin{array}{l}\text { Existência do Conselho Municipal de Meio Ambiente (CMMA). Esta variável é representada por uma dummy, } \\
\text { que recebe valor unitário caso exista o conselho no município e, zero, caso contrário. }\end{array}$ \\
\hline leg & $\begin{array}{l}\text { Existência de legislação específica para o ambiente. Esta variável é representada por uma dummy, que recebe } \\
\text { valor unitário caso exista a legislação no município e, zero, caso contrário. }\end{array}$ \\
\hline urb & $\begin{array}{l}\text { Taxa de urbanização, que corresponde à razão do número total de pessoas residentes em área urbana do } \\
\text { município e a sua população total. }\end{array}$ \\
\hline emp & $\begin{array}{l}\text { Taxa de emprego no setor formal, corresponde ao número de empregados no setor formal, em } 31 \text { de dezem- } \\
\text { bro, dividido pela população na faixa etária de } 16 \text { a } 64 \text { anos, em percentual. }\end{array}$ \\
\hline $\mathrm{pbf}$ & Número médio mensal de famílias beneficiadas com transferências do Programa Bolsa Família (PBF) no ano. \\
\hline psf & $\begin{array}{l}\text { Proporção da população atendida pelo Programa Saúde da Família (PSF), representando a cobertura do } \\
\text { programa no município. }\end{array}$ \\
\hline ete_50 & $\begin{array}{l}\text { Município com mais de } 50 \% \text { da população urbana atendida por estação de tratamento de esgoto. Esta } \\
\text { variável é representada por uma dummy, que recebe valor unitário caso o município atenda este requisito e, } \\
\text { zero, caso contrário. }\end{array}$ \\
\hline tratlix_70 & $\begin{array}{l}\text { Município com mais de } 70 \% \text { da população com acesso ao sistema de coleta de lixo tratado por meio de } \\
\text { sistema de usina de compostagem e/ou aterro sanitário. Esta variável é representada por uma dummy, que } \\
\text { recebe valor unitário caso o município atenda este requisito e, zero, caso contrário. }\end{array}$ \\
\hline
\end{tabular}

Fontes: FJP (2013) e IBGE (2013).

Elaboração das autoras.

A partir da regressão probabilística - equação (2) -, calcula-se um escore de propensão (propensity score) para cada município (i) da amostra e o pareamento será entre os municípios do grupo de controle e tratados com os valores mais similares.

Para realizar esse pareamento, existem diferentes critérios: o do vizinho mais próximo (nearest-neighbor), em que cada unidade do grupo de tratamento é pareada com a unidade do grupo de controle que possui o escore de propensão mais próximo; o pareamento radial (radius), em que o pareamento entre as unidades do grupo de controle e tratados só ocorrerá se o escore de propensão da unidade pertencente ao grupo de controle estiver em um raio pré-definido; o pareamento de Kernel, que utiliza a média ponderada de todas as unidades do grupo de controle para construir o contrafactual e os pesos utilizados são inversamente proporcionais à distância entre os valores do escore de propensão dos tratados e dos não tratados; e o estratificado (stratification), que consiste na divisão da distribuição do escore de propensão em intervalos, de modo que as unidades tratadas e de controle possuam, em média, o mesmo escore de propensão em cada intervalo (Becker e Ichino, 2002). 
Após o pareamento, é possível mensurar o efeito do tratamento (ATT), por meio da média da diferença entre os resultados $\left(Y_{i}\right)$ das unidades tratadas (municípios que implementaram a política, $D_{i}=1$ ) e das respectivas unidades de controle (municípios que não a implementaram, $D_{i}=0$ ), conforme segue:

$$
\begin{aligned}
& \mathrm{ATT}=E\left[Y_{1 i}-Y_{0 i} \mid D_{i}=1\right] \\
& A T T=E\left\{E\left[Y_{i} \mid P\left(X_{i}\right), D_{i}=1\right]-E\left[Y_{i} \mid P\left(X_{i}\right), D_{i}=0\right] \mid D_{i}=1\right\} .
\end{aligned}
$$

Ressalta-se que duas hipóteses são necessárias para obter este efeito:

- hipótese de independência condicional (HIC):

$$
P\left(X_{i}\right) \equiv E\left[D_{i} \mid X_{i}\right]=P\left[D_{i}=1 \mid X_{i}\right]
$$

Ou seja, dado um vetor de características observáveis (X), que são independentes do tratamento, os resultados potenciais serão independentes da variável binária. Isto é, as características observáveis que determinam o tratamento;

- hipótese de balanceamento:

$$
D \perp X \mid P(X) .
$$

Essa hipótese, quando satisfeita, garante que cada valor do escore de propensão, independentemente de pertencer ao grupo tratado ou de controle, tenha distribuição semelhante em relação às características observáveis (Lee, 2006).

Dessa forma, o PSM pode fornecer uma boa estimação do efeito de uma política, à medida que reduz ao máximo o viés de seleção decorrente de características não observáveis. Ressalta-se, então, a importância da seleção das características que compóem o vetor $\mathrm{X}$, pois quanto maior a qualidade destas variáveis de controle, menor será o viés de seleção.

\subsection{Base de dados e procedimento proposto}

As informaçóes utilizadas neste trabalho foram obtidas no banco de dados do Instituto Brasileiro de Geografia e Estatística (IBGE) e da Fundação João Pinheiro (FJP) e são referentes a 2013, ${ }^{4}$ ano com disponibilidade de dados mais recente. A unidade de análise desta pesquisa foi os municípios do estado de Minas Gerais.

Inicialmente, foi estimado o modelo probit para cada município - equação (2). Os resultados obtidos desta regressão foram utilizados para estimar o propensity score e realizar o pareamento.

4. Esse ano foi o último, até a realização desta pesquisa, que o IBGE identificou quais municípios implementaram o PMGIRS de acordo com as exigências da PNRS. Como se trata de uma variável essencial para a avaliação da política, essa informação acabou determinando o período de análise. 
As variáveis utilizadas nessa etapa, apresentadas no quadro 1 , foram selecionadas pois, de alguma forma, podem influenciar as variáveis de interesse e a participação do município na PNRS. E, conforme Ravallion (2007), as variáveis escolhidas para compor a regressão devem influenciar a participação no tratamento e, de alguma forma, se relacionarem com as variáveis de resultado, isto é, com os impactos que se deseja mensurar. Para isso, ainda conforme o autor, consideram-se os fatores econômicos, sociais e políticos do programa e do contexto em que está inserido.

Posteriormente, a partir de três regressóes estimadas por meio da metodologia escolhida (PSM), identificou-se o efeito médio de tratamento com relação às variáveis de resultado: existência de lixões, número de famílias vulneráveis no município e taxa de internaçóes relacionadas ao saneamento básico inadequado. Ou seja, ao estimar a equação (3) para cada uma destas variáveis, foi possível identificar o impacto da política sobre elas. A descrição das variáveis de resultado é apresentada no quadro 2 .

QUADRO 2

Descrição das variáveis resultado

\begin{tabular}{|l|l|}
\hline \multicolumn{1}{|c|}{ Variáveis } & \multicolumn{1}{c|}{ Descrição } \\
\hline vul & $\begin{array}{l}\text { Famílias vulneráveis, que corresponde ao número de famílias cuja renda mensal per capita é igual ou } \\
\text { inferior a } \mathrm{R} \$ 140,00 .\end{array}$ \\
\hline inter & $\begin{array}{l}\text { Proporção de internações por doenças relacionadas ao saneamento ambiental inadequado, que } \\
\text { consiste na razão entre o número de internações por doenças relacionadas ao saneamento ambiental } \\
\text { inadequado e o número de internações da população residente, em percentual. }\end{array}$ \\
\hline lix & $\begin{array}{l}\text { Existência de lixões no município; esta variável é representada por uma dummy, que recebe valor } \\
\text { unitário caso exista lixão no município e, zero, caso contrário. }\end{array}$ \\
\hline
\end{tabular}

Fontes: FJP (2013) e IBGE (2013).

Elaboração das autoras.

\section{RESULTADOS}

Nesta seção, são apresentados, em três tópicos, os resultados obtidos a partir da pesquisa realizada. Inicialmente, é feita uma análise descritiva da amostra; em seguida, apresenta-se o modelo probit utilizado para estimar os escores de propensão (propensity score); e, na subseção 4.3, os efeitos de tratamento são examinados.

\subsection{Descrição da amostra}

De acordo com os dados da FJP (2013) e do IBGE (2013) apresentados na tabela 1, é possível observar que, dos 853 municípios mineiros, apenas 165 possuíam a PMGIRS em 2013, ou seja, aproximadamente 19\% dos municípios mineiros implementaram o plano de acordo com as exigências da PNRS. Outras características dos municípios que compóem a amostra são: $61 \%$ possuem legislação específica 
para o meio ambiente e $77 \%$ possuem conselho municipal de meio ambiente. Além disso, $10 \%$ dos municípios mineiros possuem mais de $50 \%$ da população urbana atendida por estação de tratamento de esgoto e $24 \%$ possuem mais de $70 \%$ dos habitantes com acesso ao sistema de coleta de lixo tratado por meio do sistema de usina de compostagem e/ou aterro sanitário.

TABELA 1

Frequência das variáveis dummies do modelo probit

\begin{tabular}{|c|c|c|c|}
\hline \multicolumn{2}{|c|}{ Especificação } & \multirow{2}{*}{$\frac{\text { Frequência }}{165}$} & \multirow{2}{*}{$\frac{(\%)}{0,19}$} \\
\hline & Possui & & \\
\hline & Não possui & 688 & 0,81 \\
\hline \multirow{2}{*}{ leg } & Possui & 524 & 0,61 \\
\hline & Não possui & 329 & 0,39 \\
\hline \multirow{2}{*}{ cmma } & Possui & 657 & 0,77 \\
\hline & Não possui & 196 & 0,23 \\
\hline \multirow{2}{*}{ ete_50 } & Possui & 89 & 0,10 \\
\hline & Não possui & 764 & 0,90 \\
\hline \multirow{2}{*}{ tratlix_70 } & Possui & 205 & 0,24 \\
\hline & Não possui & 648 & 0,76 \\
\hline
\end{tabular}

Fontes: FJP (2013) e IBGE (2013).

Elaboração das autoras.

$\mathrm{Na}$ tabela 2, são apresentados os valores máximos e mínimos, a média e o coeficiente de variação de cada variável utilizada no modelo probit. Observa-se que os municípios mineiros possuem, em média, uma população de 24.142,27 habitantes e um PIB per capita de R \$ 15.296,97. Em média, 70\% da população dos municípios da amostra é urbana.

Esses dados demonstram que, embora pertençam ao mesmo estado, os municípios são heterogêneos. Nota-se que a menor dispersão é com relação ao percentual de população atendida pelo $\operatorname{PSF}(20,98 \%)$ e à taxa de urbanização $(26,25 \%)$. As variáveis população e número médio mensal de famílias beneficiadas pelo $\mathrm{PBF}$ são as que obtiveram maiores dispersóes: $407,21 \%$ e $230,31 \%$, respectivamente.

Nesse sentido, ressalta-se a importância do pareamento a fim de comparar municípios os mais semelhantes possíveis e, consequentemente, identificar de fato o impacto da política. Caso esse pareamento não seja realizado, é possível que os resultados obtidos sejam equivocados, tendo em vista que podem ser causados por outras características que não foram observadas, e não pela política em si. 
TABELA 2

Estatística básica das variáveis contínuas utilizadas no modelo probit

\begin{tabular}{lrrrc}
\hline Especificação & \multicolumn{1}{c}{ Média } & Máximo & \multicolumn{1}{c}{ Mínimo } & \multicolumn{1}{c}{$\begin{array}{c}\text { Coeficiente de } \\
\text { variação } \\
(\%)\end{array}$} \\
\hline pbf & $1.365,61$ & $71.091,00$ & 32,00 & 230,31 \\
emp & 21,70 & 100,00 & 4,00 & 56,64 \\
psf & 87,25 & 100,00 & 0,00 & 20,98 \\
pop & $24.142,27$ & $2.479 .165,00$ & 825,00 & 407,21 \\
urb & 70,03 & 100,00 & 23,06 & 26,25 \\
pibpc & $15.296,97$ & $340.144,74$ & $4.180,54$ & 121,56 \\
\hline
\end{tabular}

Fontes: FJP (2013) e IBGE (2013).

Elaboração das autoras.

\subsection{0 modelo probit}

Os resultados da regressão probit estimada são apresentados na tabela 3. A partir dessa regressão, foi possível criar o contrafactual - grupo de controle o mais semelhante possível do grupo de tratados.

As variáveis da regressão foram selecionadas de maneira que satisfaçam a hipótese de balanceamento (balancing hypothesis) e, de alguma forma, influenciem a implementação da política. Observa-se que as variáveis: existência de legislação específica para o ambiente (leg); taxa de emprego no setor formal (emp); PIB per capita $(p i b p c)$ e percentual superior a $50 \%$ da população atendida por estação de tratamento de esgoto (ete_50) foram estatisticamente significativas, considerando-se um intervalo de confiança de $0,1 \%$.

Cabe destacar que, conforme Caliendo e Kopeinig (2005), a inclusão de variáveis não significativas não torna as estimativas inconsistentes ou viesadas. Deste modo, tais variáveis só devem ser excluídas do modelo caso haja um consenso de que não sejam apropriadas (Rubin e Thomas, 1996, apud Caliendo e Kopeining, 2005).

Ao analisar os sinais apresentados pelas variáveis de controle no modelo estimado, nota-se que a existência de legislação específica para o meio ambiente, a taxa de emprego no setor formal e os municípios com mais de $50 \%$ da população atendida por estação de tratamento de esgoto têm maior probabilidade de implementar a política. 
TABELA 3

0 modelo probit: coeficientes estimados

\begin{tabular}{lcccccc}
\hline Variáveis & Coeficientes & $\begin{array}{c}\text { Erro-padrão } \\
\text { (robusto) }\end{array}$ & $z$ & $P>|z z|$ & \multicolumn{2}{c}{ Intervalo de confiança de $95 \%$} \\
\hline leg & 0,33266 & 0,11275 & 2,95 & 0,003 & 0,11168 & 0,55363 \\
pbf & 0,00002 & 0,00005 & 0,36 & 0,719 & $-0,00007$ & 0,00011 \\
emp & 0,01250 & 0,00649 & 1,93 & 0,054 & $-0,00021$ & 0,02522 \\
psf & $-0,00150$ & 0,00288 & $-0,52$ & 0,603 & $-0,00715$ & 0,00415 \\
cmma & 0,18307 & 0,13365 & 1,37 & 0,171 & $-0,07888$ & 0,44502 \\
pop & $-1,81 \mathrm{e}-07$ & $1,52 \mathrm{e}-06$ & $-0,12$ & 0,905 & $-3,16 \mathrm{e}-06$ & $2,80 \mathrm{e}-06$ \\
urb & 0,00450 & 0,00345 & 1,30 & 0,192 & $-0,00226$ & 0,01127 \\
pibpc & $-0,00002$ & $6,14 \mathrm{e}-06$ & $-2,37$ & 0,018 & $-0,00003$ & $-2,51 \mathrm{e}-06$ \\
ete_50 & 0,36818 & 0,15791 & 2,33 & 0,020 & 0,05868 & 0,67767 \\
tratlix_70 & 0,01869 & 0,12315 & 0,15 & 0,879 & $-0,22267$ & 0,26005 \\
_cons & $-1,55618$ & 0,39906 & $-3,90$ & 0,000 & $-2,33832$ & $-0,77404$ \\
\hline
\end{tabular}

Fonte: Resultados da pesquisa.

Elaboração das autoras.

Obs.: Variável dependente: dummy igual a um para os municípios que implementaram a PMGIRS e zero, caso contrário. Número de observações: 853.

A partir da estimação do modelo probit, foi possível dividir os municípios em grupos, de acordo com a probabilidade de implementar a política obtida por cada um, dadas as características observáveis. Ou seja, a partir da probabilidade de implementação da política de cada município, foi realizado o pareamento entre os grupos de tratados e controle. Na tabela 4, é possível verificar o limite inferior, os números de tratados e de controles de cada bloco, considerando o suporte comum. Nota-se que, dos 853 municípios, apenas 24 não foram pareados, por falta de suporte comum.

TABELA 4

Limite inferior, números de controles e de tratamentos para cada bloco

\begin{tabular}{lrcc}
\hline $\begin{array}{l}\text { Limite inferior do escore de propensão } \\
\text { (suporte comum) }\end{array}$ & Controle & Tratamento & Total \\
\hline 0,0719691 & 70 & 9 & 79 \\
0,1 & 169 & 17 & 186 \\
0,15 & 160 & 45 & 205 \\
0,2 & 256 & 85 & 341 \\
0,4 & 9 & 8 & 17 \\
0,8 & 0 & 1 & 1 \\
Total & 664 & 165 & 829 \\
\hline
\end{tabular}

Fonte: Resultados da pesquisa.

Elaboração das autoras. 
A tabela 5 apresenta o teste de médias das variáveis utilizadas para estimação do modelo. Observa-se que, antes do pareamento, existia uma diferença entre os grupos de tratado e controle em relação à média da maioria das variáveis. Somente o PIB per capita ( $p i b p c)$ e o acesso ao sistema de coleta de lixo tratado por meio de sistema de usina de compostagem e/ou aterro sanitário para mais de $70 \%$ da população (tratlix_70) não eram estatisticamente diferentes entre estes grupos. Após o pareamento, a diferença das médias entre os dois grupos foi estatisticamente não significativa para todas as variáveis, o que demonstra a qualidade do pareamento realizado.

TABELA 5

Teste de médias antes e depois do pareamento

\begin{tabular}{|c|c|c|c|c|}
\hline \multirow{2}{*}{ Variáveis } & & \multicolumn{2}{|c|}{ Média } & \multirow{2}{*}{$\begin{array}{l}\text { Teste de média } \\
\text { (p-valor) }\end{array}$} \\
\hline & & Tratamento & Controle & \\
\hline \multirow{2}{*}{ leg } & NP & 0,746 & 0,583 & 0,000 \\
\hline & $P$ & 0,739 & 0,733 & 0,900 \\
\hline \multirow{2}{*}{$\mathrm{pbf}$} & NP & 1.928 & $1.230,7$ & 0,010 \\
\hline & $P$ & $1.376,8$ & $1.396,3$ & 0,940 \\
\hline \multirow{2}{*}{ emp } & NP & 23,878 & 21,474 & 0,034 \\
\hline & P & 23,183 & 23,229 & 0,972 \\
\hline \multirow{2}{*}{ psf } & NP & 84,675 & 87,865 & 0,044 \\
\hline & $P$ & 84,706 & 84,874 & 0,941 \\
\hline \multirow{2}{*}{ cmma } & NP & 0,842 & 0,753 & 0,014 \\
\hline & P & 0,839 & 0,817 & 0,607 \\
\hline \multirow{2}{*}{ pop } & NP & 40.658 & 20.181 & 0,016 \\
\hline & $P$ & 23.787 & 28.149 & 0,548 \\
\hline \multirow{2}{*}{ urb } & NP & 73,606 & 69,186 & 0,006 \\
\hline & $P$ & 73,202 & 72,777 & 0,828 \\
\hline \multirow{2}{*}{ pibpc } & NP & 14.493 & 15.342 & 0,599 \\
\hline & $P$ & 14.342 & 14.040 & 0,791 \\
\hline \multirow{2}{*}{ ete_50 } & NP & 0,176 & 0,087 & 0,001 \\
\hline & $P$ & 0,155 & 0,165 & 0,820 \\
\hline \multirow{2}{*}{ tratlix_70 } & NP & 0,242 & 0,240 & 0,944 \\
\hline & P & 0,249 & 0,261 & 0,799 \\
\hline
\end{tabular}

Fonte: Resultados da pesquisa.

Elaboração das autoras.

Obs.: NP - não pareados; $\mathrm{P}$ - pareados. 


\subsection{Efeito e impactos da política}

Nas tabelas 6, 7 e 8, são apresentados, respectivamente, os impactos da política sobre a existência de lixóes nos municípios, sobre o número de famílias vulneráveis e sobre a proporção de internaçóes decorrentes de saneamento básico inadequado.

A análise dos impactos foi feita com base nos seguintes pontos: valor estimado e sinal do efeito médio de tratamento nos tratados (ATT); e significância estatística (T). Foram utilizados diferentes critérios de pareamento (matching): vizinho mais próximo, radial, estratificado e kernel, a fim de obter maior robustez nos resultados.

Considerando que um dos objetivos da política é eliminar os lixões em todo o país, buscou-se avaliar o efeito da política sobre a existência de lixóes nos municípios mineiros. Como se pode observar na tabela 6 , apesar de o $A T T$ ter sido negativo em todos os modelos de pareamento utilizados, o que indicaria que a existência de lixões seria menor nos municípios tratados, esse resultado não foi estatisticamente significativo. Ou seja, não há diferença entre os municípios que compóem o grupo de tratados e de controle em relaçẫo à presença de lixóes.

É válido lembrar que o estado de Minas Gerais implementou em 2003 o programa Minas sem Lixóes, ${ }^{5}$ cujo principal objetivo é a erradicação dos lixões nos municípios mineiros. Segundo Magalhães e Ribeiro (2017), desde sua implementação até 2013, o número de municípios que possuíam lixôes reduziu de 823 para 264, sendo que, em 2010, quando a PNRS foi implementada, esse número era de 311 .

Note que, embora ainda tenha reduzido o número de lixóes no estado após a implementação da PNRS, a redução mais significativa (aproximadamente 62\%) foi entre 2003 e 2010. Ressalta-se que isso não significa que a PNRS não tenha apresentado eficácia em relaçáo à erradicação dos lixốes ou que o programa Minas sem Lixóes tenha reduzido sua efetividade nos últimos anos. Mas sim que é necessário um tempo maior para que um lixão seja encerrado.

De acordo com Lins (2017), não há registro de encerramento de lixões e implantação de aterros sanitários em um prazo inferior a quatro anos em nenhum país do mundo. Além disso, segundo a Associação Brasileira de Empresas de Tratamento de Resíduos (Abetre), o custo total para a viabilização de um aterro sanitário, necessário para substituir o lixão encerrado, está entre $\mathrm{R}$ \$2,4 milhóes e R \$ 535,8 milhôes, dependendo do seu porte (Abetre, [s.d.]).

Todos esses fatores podem ter influenciado o resultado não significativo da PNRS em relação à existência de lixóes.

5. Esse programa foi criado pela Fundação Estadual do Meio Ambiente (Feam) para auxiliar os municípios do estado na gestão adequada dos RSUs, conforme normas estabelecidas pelo Conselho Estadual de Política Ambiental - Copam (Magalhães e Ribeiro, 2017). 
TABELA 6

Impacto da política sobre a existência de lixões

\begin{tabular}{lccccc}
\hline & Tratados & Controle & ATT & Erro padrão & $T^{1}$ \\
\hline $\begin{array}{l}\text { Vizinho mais } \\
\text { próximo }\end{array}$ & 165 & 133 & $-0,018$ & 0,066 & $-0,278$ \\
Radial & 163 & 664 & $-0,024$ & 0,043 & $-0,562$ \\
Estratificado & 164 & 665 & $-0,017$ & 0,051 & $-0,323$ \\
Kernel & 165 & 664 & $-0,018$ & 0,039 & $-0,451$ \\
\hline
\end{tabular}

Fonte: Resultados da pesquisa.

Elaboração das autoras.

Nota: ${ }^{1} A$ estatística $t$ é baseada no erro padrão calculado por meio do método bootstrap (cinquenta repetições).

Com relação ao número de famílias vulneráveis, verifica-se, na tabela 7, que a política também não teve impacto estatisticamente significativo em nenhum dos métodos de pareamento utilizado. Ou seja, embora a política tenha como meta incentivar a inclusão social dos catadores, melhorando as condiçóes de trabalho e formalizando o trabalho realizado por meio de cooperativas, o que elevaria a renda destes trabalhadores, isso ainda não teve impacto sobre o número de famílias vulneráveis.

Segundo Demajorovic et al. (2014), muitas cooperativas enfrentam problemas organizacionais e operacionais, náo possuindo uma estrutura adequada, comprometendo assim seus ganhos, o que pode justificar o impacto estatisticamente não significativo do programa sobre o número de famílias vulneráveis. Neste sentido, os autores destacam a importância das parcerias entre catadores/cooperativas, organizaçóes não governamentais (ONGs) e setores público e privado para superar estas limitaçóes.

Cabe ainda destacar que as famílias vulneráveis da amostra analisada não se referem apenas aos catadores, mas a todas as famílias que se encontram em vulnerabilidade. Isto é, o número de famílias vulneráveis corresponde ao número de famílias do município cuja renda mensal per capita é igual ou inferior a $\mathrm{R} \$ 140,00$. Isso também pode ter influenciado o impacto não significativo da PNRS sobre essa variável. 
TABELA 7

Impacto da política sobre o número de famílias vulneráveis

\begin{tabular}{lccccc}
\hline & Tratados & Controle & ATT & Erro padrão & $T^{1}$ \\
\hline $\begin{array}{l}\text { Vizinho mais } \\
\text { próximo }\end{array}$ & 165 & 133 & 502,988 & 740,192 & 0,680 \\
Radial & 163 & 664 & 358,941 & 257,485 & 1,394 \\
Estratificado & 164 & 665 & 205,391 & 402,635 & 0,510 \\
Kernel & 165 & 664 & 864,493 & 673,221 & 1,284 \\
\hline
\end{tabular}

Fonte: Resultados da pesquisa.

Elaboração das autoras.

Nota: ${ }^{1}$ A estatística $t$ é baseada no erro padrão calculado por meio do método bootstrap (cinquenta repetições).

A tabela 8 apresenta o impacto da política sobre a proporção de internaçôes decorrentes do saneamento básico inadequado. Essa análise permite identificar o efeito da política sobre a saúde dos habitantes. A PNRS e, em consequência, a PMGIRS buscam tratar os resíduos sólidos de forma adequada, dando o destino apropriado aos produtos no fim de sua vida útil. Dessa forma, espera-se que a contaminação e a poluição do meio ambiente sejam minimizadas, resultando em melhor qualidade de vida para a população.

Observa-se, conforme os dados apresentados na tabela 8, que o efeito médio $(A T T)$ da política sobre a proporção de internaçôes causadas por saneamento básico inadequado é negativo em todos os métodos de pareamento utilizados. $\mathrm{Ou}$ seja, a proporção de internaçôes é menor nos municípios que implementaram a PMGIRS, trazendo benefícios à saúde de seus habitantes.

É importante destacar a possibilidade de outros fatores como taxa de urbanização municipal, cobertura do PSF no município, percentual da população atendida por tratamento de esgoto e com acesso ao sistema de coleta de lixo por meio de sistema de usina de compostagem e/ou aterro sanitário influenciarem a proporção de internaçôes causadas pelo saneamento inadequado.

Apesar disso, a metodologia utilizada considerou todas essas características na construção do contrafactual, fazendo com que a comparação fosse feita entre municípios os mais semelhantes possível. Assim, ao considerar todos esses fatores como variáveis que poderiam afetar, de alguma forma, o percentual de internaçóes nos municípios, o PSM fornece uma boa estimação do efeito da política.

Os métodos vizinhos mais próximos, radial e Kernel, foram estatisticamente significativos a $5 \%$, demonstrando que os municípios que implementaram a política possuem uma proporção desse tipo de internação em torno de 4,896 pontos percentuais (p.p.); 1,861 p.p. e 2,003 p.p. menores que os municípios que não a implementaram, respectivamente. O método estratificado foi significativo a $10 \%$, 
revelando que municípios tratados possuem, em média, uma proporção de internaçóes devido ao saneamento inadequado 1,988 p.p. inferior à dos municípios de controle.

Dado que a proporção de internaçóes decorrentes de saneamento básico inadequado dos municípios da amostra é, em média, 4,912 p.p. e que há uma redução de, no mínimo, 1,861 p.p. nesta proporção nos municípios tratados, isso equivale a uma redução de, aproximadamente, $38 \%$ deste tipo de internação nestes municípios. Cabe destacar que este impacto é de suma relevância, haja vista que, por meio dos benefícios à saúde, há uma melhora na qualidade de vida da população podendo, inclusive, impactar a formação de capital humano nestas regióes.

Conforme Todaro e Smith (2009), melhores condições de educação e saúde impactam positivamente a formação de capital humano, aumentando a produtividade e, consequentemente, o desenvolvimento da região. Cabe salientar que, para os autores, o investimento em capital humano é essencial para o desenvolvimento.

De outra maneira, além de uma melhor qualidade de vida, os impactos de uma populaçáo mais "saudável" sáo de longo prazo, podendo, inclusive, influenciar significativamente o desenvolvimento regional.

TABELA 8

Impacto da política sobre a proporção de internações decorrentes de saneamento básico inadequado

\begin{tabular}{lccccc}
\hline & Tratados & Controle & ATT & Erro-padrão & $T^{1}$ \\
\hline Vizinho mais próximo** $^{*}$ & 165 & 133 & $-4,896$ & 2,149 & $-2,278$ \\
Radial** $^{*}$ & 163 & 664 & $-1,861$ & 0,938 & $-1,984$ \\
Estratificado* $^{*}$ & 164 & 665 & $-1,988$ & 1,140 & $-1,745$ \\
Kernel** $^{*}$ & 165 & 664 & $-2,003$ & 0,984 & $-2,036$ \\
\hline
\end{tabular}

Fonte: Resultados da pesquisa.

Elaboração das autoras.

Nota: ${ }^{1}$ A estatística $t$ é baseada no erro-padrão calculado por meio do método bootstrap (cinquenta repetições).

Obs.: * Significativo a 10\%. * ${ }^{*}$ Significativo a $5 \%$.

Os resultados obtidos revelam que os impactos de uma política pública podem ocorrer em períodos distintos, isto é, o tempo de maturação da política influencia o prazo de alcance dos objetivos propostos em sua formulação e implementação. O objetivo da PNRS é dar tratamento adequado aos resíduos sólidos, eliminando os lixóes e incentivando a coleta seletiva, por exemplo. Enquanto os resultados referentes à existência de lixóes náo foram significantes estatisticamente, revelando que a política não havia trazido efeito até 2013 , ano-base dos dados analisados, em relação a esse objetivo; o resultado sobre a variável internações foi estatisticamente significativo. 
Nesse sentido, a política obteve um impacto de suma importância para a população, pois os municípios que a implementaram possuem menor proporção de internações causadas pelo saneamento básico inadequado, indicando melhores condiçóes de saúde e, consequentemente, melhor qualidade de vida da população.

Resultados semelhantes foram encontrados por Okawara (2018) que, ao avaliar os possíveis impactos de políticas de resíduos sólidos na saúde, verificou que a implementação da PMGIRS pode reduzir os casos de leptospirose em municípios com menos de 11 mil habitantes.

Vale ressaltar que esse impacto pode trazer outros resultados benéficos para toda a sociedade, como o aumento da produtividade e a formação de capital humano, fatores essenciais para o desenvolvimento.

Desse modo, a partir deste trabalho, é possível verificar que, ao avaliar uma política pública, é importante considerar não apenas os objetivos propostos no momento de sua formulação, mas também o prazo de maturação da política, haja vista a necessidade de maior tempo para alcançar determinados objetivos. Neste estudo, embora a eliminação de lixōes, representada pela variável existência de lixôes, seja um dos objetivos da PNRS, o efeito da política sobre essa variável não foi significante estatisticamente.

Observou-se que a política trouxe benefícios relacionados à saúde, em conformidade com seus objetivos, tendo em vista que a proporção de internaçóes causadas pelo mau saneamento básico nos municípios tratados é menor que nos municípios de controle. Deste modo, pode-se concluir que a política trouxe impactos positivos para os municípios que a implementaram.

Conclui-se, então, que a PNRS trouxe impactos positivos para o bem-estar e a qualidade de vida da população por meio dos benefícios causados à saúde. Ou seja, os ganhos advindos da política, embora num período relativamente curto, foram significativos e relevantes para a sociedade. Sendo assim, é importante destacar a complexidade de se avaliar políticas públicas e a importância de se considerar diversos fatores como o tempo de maturação nesta etapa. Isto é, uma análise cautelosa e imparcial ao avaliar a eficácia de determinada política é necessária.

\section{CONSIDERAÇÕES FINAIS}

As questôes ambientais estáo cada vez mais em destaque, sendo discutidas por governantes de inúmeros países, desenvolvidos ou em desenvolvimento. Neste sentido, há uma busca contínua por formas de manter o crescimento econômico dos países, minimizando os danos causados ao meio ambiente. 
A PNRS, implementada em 2010, por meio da Lei no $12.305 / 2010$, é uma das consequências desta demanda. Vale ressaltar, que se trata de uma reivindicação recorrente, tendo em vista que existe desde 1991.

Considerando a importância das questôes ambientais e que a política tem como um de seus objetivos dar o tratamento e o destino adequados para os resíduos sólidos, buscou-se avaliar os impactos da política nos municípios mineiros que implementaram a PMGIRS, conforme exigências da PNRS. Os resultados obtidos revelam que esses municípios possuem uma proporção menor de internaçóes relacionadas ao saneamento básico inadequado, demonstrando que a política tem trazido impactos significativos na saúde da população mineira.

Vale ressaltar que, para o encerramento dos lixóes, é necessário um planejamento adequado e um prazo maior para a criação de alternativas, como aterros sanitários. Além disso, os investimentos necessários para realizar uma gestão adequada dos resíduos sólidos são elevados, indo de encontro à realidade financeira de muitos municípios. Isso pode justificar os resultados não significativos referentes a esta variável.

A PNRS, então, embora ainda não tenha atingido alguns de seus objetivos, como a eliminação de lixóes, obteve na amostra estudada efeitos de suma importância para o desenvolvimento da sociedade, haja vista que benefícios gerados à saúde elevam a qualidade de vida da população e impactam positivamente a formação de capital humano.

Nesse contexto, observa-se a importância de se avaliar as políticas públicas de forma imparcial, considerando os diversos impactos que a política pode gerar e o seu prazo de maturaçáo. Sendo assim, deve-se ter cautela ao decretar que determinada política não obteve sucesso, tendo em vista que seus efeitos podem ir muito além dos objetivos propostos nas fases de formulação e implementação.

Ressalta-se que este estudo, devido à indisponibilidade de dados, analisou um período relativamente curto desde a implementação da política, o que pode justificar alguns resultados estatisticamente não significativos. Mais uma vez, destaca-se a importância de informações referentes à efetiva implementação do PMGIRS em atendimento à PNRS e de dados relacionados aos RSUs para que estudos complementares, com dados mais recentes, sejam realizados.

\section{REFERÊNCIAS}

ABETRE - ASSOCIAÇÃO BRASILEIRA DE EMPRESAS DE TRATAMENTO DE RESÍDUOS. Estudo sobre os aspectos econômicos e financeiros da implantação e operaçáo de aterros sanitários. [s.l.]: Abetre, [s.d.]. Disponível em: <https://bit.ly/33A97z1>. Acesso em: 18 abr. 2018. 
ALENCAR, T. S.; ROCHA, J. P. M.; SILVA, R. V. Política nacional de resíduos sólidos e os catadores de materiais recicláveis: estudo de caso de cooperativas nos municípios do estado do Rio de Janeiro. Revista Nacional de Gerenciamento de Cidades., v. 3, n. 20, p. 34-52, 2015.

ANDERSON, C. W. The place of principles in policy analysis. American Political Science Review, v. 73, n. 3, p. 711-723, Sept. 1979.

ARCILA, R. I. A. Panorama dos resíduos sólidos urbanos nos municípios de pequeno porte do Brasil. $58 \mathrm{f}$. Dissertação (Mestrado) - Curso de desenvolvimento em meio ambiente, Universidade Federal do Rio Grande do Norte, Natal, 2008.

BECKER S. O.; ICHINO, A. Estimation of average treatment effects based on propensity score. Stata Journal, v. 2, n. 4, p. 358-377, 2002.

BESEN, G. R. et al. Resíduos sólidos: vulnerabilidades e perspectivas. In: SALDIVA, P. et al. Meio ambiente e saúde: o desafio das metrópoles. São Paulo: Ex Libris, 2010.

BRASIL. Projeto de Lei no 203/1991, de 1o de abril de 1991. Institui a Política Nacional de Resíduos Sólidos; altera a Lei no 9.605, de 12 de fevereiro de 1998; e dá outras providências. Diário Oficial da Uniáa, Brasília, 1a abr. 1991. Disponível em: <https://bit.ly/2KLMBN1>. Acesso em: 25 nov. 2017.

. Projeto de Lei no 1991/2007, de 11 de julho de 2007. Institui a Política Nacional de Resíduos Sólidos e dá outras providências. Diário Oficial da Uniáo, Brasília, 11 jul. 2007. Disponível em: <https://bit.ly/36sdKgg>. Acesso em: 25 nov. 2017.

Lei no 12.305, de 2 de agosto de 2010. Institui a Política Nacional de Resíduos Sólidos; altera a Lei oํ 9.605, de 12 de fevereiro de 1998; e dá outras providências. Diário Oficial da Uniáo, Brasília, 2 ago. 2010a. Disponível em: <www.planalto.gov.br>. Acesso em: 10 nov. 2017.

. Decreto no 7.404 , de 23 de dezembro de 2010. Regulamenta a Lei no 12.305, de 2 de agosto de 2010, que institui a Política Nacional de Resíduos Sólidos, cria o Comitê Interministerial da Política Nacional de Resíduos Sólidos e o Comitê Orientador para a Implantação dos Sistemas de Logística Reversa, e dá outras providências. Diário Oficial da Uniáo, Brasília, 23 dez. 2010b. Disponível em: <www.planalto.gov.br>. Acesso em: 10 nov. 2017.

. Ministério do Meio Ambiente (MMA). Contexto e principais aspectos. [s.l.]: MMA, [s.d.]. Disponível em: <https://bit.ly/3fSKJ0F>. Acesso em: 7 nov. 2017. 
CALIENDO, M.; KOPEINIG, S. Some practical guidance for the implementation of propensity score matching. Bonn, Germany: IZA, 2005. (Discussion papers, n. 1588).

CASTRO, M. A. O. Avaliação dos sistemas de gestão de resíduos sólidos nos municípios de Iranduba, Manacapuru e Novo Airáo, AM. 142 f. Dissertação (Mestrado) - Programa de Pós-graduação em Ciências do Ambiente e Sustentabilidade, Universidade Federal do Amazonas, Manaus, 2012.

CASTRO, A. M. R. C.; SOUZA, N. D.; ALBINO, P. M. B. Avaliaçáo do impacto da política nacional de resíduos sólidos na coleta seletiva em Viçosa-MG. In: FÓRUM INTERNACIONAL DE RESÍDUOS SÓLIDOS, 8., 2017, Curitiba, Paraná. Anais... Curitiba: FIRS, 2017.

COBB, R.; ELDER, C. Participation in american politics: the dynamics of agenda-building. Baltimore: Johns Hopkins University Press, 1983.

COSTA, F. L.; CASTANHAR, J. C. Avaliação de programas públicos: desafios conceituais e metodológicos. Revista de Administraçáo Pública, Rio de Janeiro, v. 37, n. 5, p. 969-992, set./out. 2003.

DEMAJOROVIC, J. et al. Integrando empresas e cooperativas de catadores em fluxos reversos de resíduos sólidos pós-consumo: o caso Vira-Lata. Caderno EBAPE. BR, Edição Especial, p. 513-532, ago. 2014.

EASTON, D. Modalidades de análise política. Rio de Janeiro: Zahar, 1970.

FARIA, C. A. P.; FILGUEIRAS, C. A. C. As políticas dos sistemas de avaliação da educação básica. In: HOCHMAN, G.; ARRETCHE, M.; MARQUES, E. (Orgs.). Políticas públicas no Brasil. Rio de Janeiro: Fiocruz, 2007. p. 327- 367.

FERREIRA, J. A.; ANJOS, L. A. Aspectos de saúde coletiva e ocupacional associados à gestáo dos resíduos sólidos municipais. Cadernos de Saúde Pública, Rio de Janeiro, v. 17, n. 3, p. 689-696, maio/jun. 2001.

FJP - FUNDAÇÃO JOÃO PINHEIRO. Índice mineiro de responsabilidade social. Belo Horizonte: FJP, 2013. Disponível em: <http://imrs.fjp.mg.gov.br>. Acesso em: 10 dez. 2017.

GIULIANI, M. Policy termination. In: CAPANO, G.; GIULIANI, M. Dizionario di politiche pubbliche. Roma: Carocci, 2005.

GOUVEIA, N. Saúde e meio ambiente nas cidades: os desafios da saúde ambiental. Saúde e Sociedade, São Paulo, v. 8, n. 1, p. 49-61, jan./fev. 1999.

GUBA, E.; LINCOLN, Y. Fourth generation evaluation. Newbury Park: Sage Publications, 1989. 
HAM, C.; HILL, M. The policy process in the modern capitalist state. London: Harvester, 1993.

IBGE - INSTITUTO BRASILEIRO DE GEOGRAFIA E ESTATÍSTICA. Perfil dos municípios brasileiros 2013. Rio de Janeiro: IBGE, 2013. Disponível em: $<$ https://bit.ly/2JyNV5c>. Acesso em: 14 nov. 2017.

JACOBI, P. R.; BESEN, G. R. Gestáo de resíduos sólidos em São Paulo: desafios da sustentabilidade. Estudos Avançados, São Paulo, v. 25, n. 71, jan./abr. 2011.

LASWELL, H. D. Politics: Who gets what, when, how. Cleveland: Meridian books, 1936.

LEE, W. Propensity score matching and variations on the balancing test. Melbourne: Melbourne Institute of Applied Economics and Social Research, 2006.

LINS, C. A gestáo de resíduos sólidos no Brasil e os desafios ao cumprimento da Lei no 12.305, de 2010, São Paulo: IEE, 2017. Disponível em: <https://bit. ly/3mn9qoe>. Acesso em: 15 abr. 2018.

LYNN, L. E. Designing public policy: a casebook on the role of policy analysis. Santa Monica, Califórnia: Goodyear, 1980.

MAGALHÃES, R. A.; RIBEIRO, K. A. S. Política estadual de gestão de resíduos: uma análise do programa "Minas sem lixóes". Revista Direito Ambiental e Sociedade, v. 7, n. 1, p. 34-61, 2017.

MEAD, L. M. Public Policy: vision, potential, limits. Policy Currents, v. 68, n. 3, fev. 1995.

MONTEIRO, J.H.P. et al. Manual de Gerenciamento Integrado de Resíduos Sólidos. Rio de Janeiro: IBAM, 2001.

NASCIMENTO, L. J. B. F. et al. Avaliaçáo dos planos de gerenciamento de resíduos sólidos em municípios com população superior a 20.000 habitantes no estado do Paraná frente a Lei no ${ }^{12.305}$. In: FÓRUM INTERNACIONAL DE RESÍDUOS SÓLIDOS, 8., 2017, Curitiba, Paraná. Anais... Curitiba: FIRS, 2017.

O`TOOLE JUNIOR, L. J. Interorganizational relations in implemention. In: PETERS, B. G.; PIERRE, J. (Orgs.). Handbook of public administration. Londres; Califórnia: Thousand oaks; Sage publications, 2003.

OKADA, D. S. Panorama da política nacional de resíduos sólidos: Lei no 12.305/2010. In: AHMED, F. Curso de direito ambiental. Rio de Janeiro: Lumen Juris, 2012. p. 135-155. 
OKAWARA, J. M. Avaliação de impacto da política de resíduos sólidos na saúde. 75 f. Dissertação (Mestrado) - Escola de Pós-Graduação em Economia, Fundação Getulio Vargas, São Paulo, 2018.

PETERS, B. G. American public policy. Chatham: Chatham House. 1986.

PINTO, I. C. M. Mudanças nas políticas públicas: a perspectiva do ciclo de políticas públicas. Revista de Políticas Públicas de Sáo Luís, v. 12, n. 1, p. 27-36, jan./jun. 2008.

RAVALLION, M. Evaluating Anti-Poverty Programs. Handbook of Development Economics, v. 4, 2007.

ROSENBAUM, P.; RUBIN, D. B. The central role of the propensity score in observational studies for causal effects. Biometrika, v. 70, n. 1, p. 41-55, 1983.

SCHATTSCHNEIDER, E. E. The semisovereign people: a realist's view of democracy in America. Nova York: Holt Rinehart and Winston, 1960.

SETTE, M. T. D.; NOGUEIRA, J. M. Política nacional de resíduos sólidos: uma avaliação inicial acerca dos aspectos jurídicos e econômicos. Revista Jurídica da Universidade de Cuiabá, v. 12, n. 2, p. 157-184, 2010.

SILVA, C. L.; FUGII, G. M.; SANTOYO, A. H. Proposta de um modelo de avaliação das açôes do poder público municipal perante as políticas de gestão de resíduos sólidos urbanos no Brasil: um estudo aplicado ao município de Curitiba. URBE. Revista Brasileira de Gestáo Urbana, v. 9, n. 2, p. 276-292, maio/ago. 2017.

SILVA, P. F.; TEIXEIRA, B. A. N. Avaliação da coleta seletiva no plano municipal de gestấo integrada de resíduos sólidos de Barreiras-BA. Revista Nacional de Gerenciamento de Cidades, v. 4, n. 25, p. 114-127, 2016.

SJOBLOM, G. Problemi e soluzioni in politica. Rivista Italiana di Scienza Politica, v. 14, n. 1, p. 41-85, abr. 1984.

SOUSA, F. H. F.; FERNANDES, A. J. M. M. A problemática dos impactos ambientais causados pelo funcionamento do lixão do município de Imperatriz-MA. In: FÓRUM INTERNACIONAL DE RESÍDUOS SÓLIDOS, 7., 2016, Rio Grande do Sul. Anais... Rio Grande do Sul: FIRS, 2016.

TODARO, M. P.; SMITH, S. C. Economic Development. 11. ed. New Jersey: Prentice Hall, 2009.

WOOLDRIDGE, J. M. Introduçáo à econometria: uma abordagem moderna. 2. ed. São Paulo: Thompson Learning, 2000.

Econometrics analysis of cross section and panel data. Cambridge:

The MIT Press, 2002. 
Data da submissão: 30/7/2018

Primeira decisão editorial em: 7/1/2019

Última versão recebida em: 13/4/2019

Aprovação final em: 26/4/2019 